\title{
Relationship of Vertigo and Postural Instability in Patients With Vestibular Schwannoma
}

\author{
Gi-Sung Nam · Chan Min Jung · Ji Hyung Kim · Eun Jin Son \\ Department of Otorhinolaryngology, Gangnam Severance Hospital, Yonsei University College of Medicine, Seoul, Korea
}

Objectives. Growth of vestibular schwannomas (VS) causes progressive vestibular symptoms and postural instability. Since the tumor grows slowly, compensation of decaying vestibular input may decrease subjective symptoms of dizziness. This study aims to estimate the relationship of subjective vestibular symptoms and objective postural instability in patients with VS.

Methods. A retrospective review of 18 patients newly diagnosed with VS and with subjective vertigo symptoms was performed. The results of vestibular function tests including the sensory organization test (SOT) using computerized dynamic posturography, caloric test, and self-report measures of subjective dizziness handicap (Dizziness Handicap Inventory) and visual analogue scale were compared according to the onset of vertigo symptoms.

Results. InVS patients, SOT showed decreased equilibrium score for all vestibular function related conditions, condition (C) 5 and 6, and composite (COMP) score. COMP scores were not correlated with visual analogue scale or Dizziness Handicap Inventory scores. Acute onset group included six patients and insidious onset group, 12 patients. Equilibrium scores for C5 and C6, and COMP scores were lower for insidious onset group, but the difference was not statistically significant.

Conclusion. Our findings confirmed postural instability is prevalent in VS patients. SOT parameters did not differ significantly between acute onset and insidious onset groups, but increased tumor size and canal weakness were noted in the insidious onset group. Clinicians should consider that postural instability is likely present even in patients who do not complain of acute vertigo, and appropriate counseling should be discussed with the patients.

Keywords. Acoustic Neuroma; Vertigo; Postural Balance; Posturography

\section{INTRODUCTION}

Vestibular schwannomas (VS) are benign tumors arising from the Schwann cells of the vestibular nerve. While vestibular symptoms are expected from the nerve of origin, auditory symptoms such as hearing loss and tinnitus are most common symptoms presenting at the time of diagnosis [1]. Patients with VS may present with vestibular symptoms of significant vari-

\footnotetext{
- Received September 21, 2017

Revised November 1, 2017

Accepted November 8, 2017

- Corresponding author: Eun Jin Son

Department of Otorhinolaryngology, Gangnam Severance Hospital, Yonsei

University College of Medicine, 211 Eonju-ro, Gangnam-gu, Seoul 06273,

Korea

Tel +82-2-2019-3460, Fax +82-2-3463-4750

E-mail: ejson@yuhs.ac
}

ability in severity and progression; symptoms include vertigo and dizziness, and sometimes postural instability [1-7]. The onset of the symptoms is variable because the central compensation may mask the development of slowly ongoing peripheral vestibular hypofunction with slow-growing tumors. Gradual deterioration of vestibular function eventually leads to postural instability, which poses significant impact on patients' quality of life $[8,9]$.

Many researches have focused on the relationship of objective parameters such as vestibular function tests and tumor characteristics to explain the wide spectrum of vertigo in VS patients. Caloric testing has been the most common method to evaluate vestibular function in VS patients, and more recently vestibular myogenic evoked potentials (VEMP) and video head impulse test (vHIT) have been employed [1,10-14]. Larger tumors have been associated with more severe caloric paresis $[12,13]$. Calor-

Copyright $@ 2018$ by Korean Society of Otorhinolaryngology-Head and Neck Surgery.

This is an open-access article distributed under the terms of the Creative Commons Attribution Non-Commercial License (http://creativecommons.org/licenses/by-nc/4.0)

which permits unrestricted non-commercial use, distribution, and reproduction in any medium, provided the original work is properly cited. 
ic weakness and vHIT abnormality were shown to correlate with tumor size, but not with vertigo symptoms in VS patients $[1,10]$. Abnormal in vHIT results were found in VS patients with normal caloric responses, suggesting conventional caloric testing may not be sensitive to vestibular dysfunction caused by VS [11]. It was reported that abnormal vHIT and caloric weakness were correlated with tumor size, especially when the large tumors were compressing the brainstem [14]. Abnormalities in VEMPs showed correlation to tumor size [4], and a large-scale study of VS patients showed that larger tumors were associated with higher risk of canal paresis and postural instability [2]. Similar to other causes of unilateral vestibular deficit, objective dysfunction measured by caloric, vHIT or VEMP in VS patients does not necessarily correlate with subjective symptom severity or progression. It has been suggested that gradual loss of the peripheral vestibular input would allow adequate central vestibular compensation to alleviate vertigo symptoms.

In addition to adequate vestibular input, visual and somatosensory information is required to maintain balance, and the computerized dynamic posturography (CDP) can provide an objective assessment of the postural stability $[6,15]$. It can be assumed that postural control will deteriorate with gradual decline of vestibular input that will likely occur with tumor growth, even if the patient does not experience severe vertigo symptoms. The VS with vertigo, dizziness and/or imbalance symptoms had lower scores in CDP testing compared with those without symptoms [16], and even patients with small tumors scored lower than normal subjects [17]. It has been reported that only $10 \%$ of the variance in subjective vertigo symptom can be explained by differences in postural stability [2]. The CDP results showed a unique correlation between tumor size and postural instability, with increased postural sway when the tumor was small, which improved with increased tumor size, then again increased postural sway with even larger size of the tumor compressing the brainstem [17].

Although postural instability in VS patients has been described with respect to patient and tumor characteristics, to our knowledge, there are few reports to correlate the acuteness of

\section{H I G G H L I G G H T S}

- Postural instability is prevalent in vestibular schwannoma (VS) patients with acute onset and insidious onset vestibular symptoms.

- Computerized dynamic posturography parameters were correlated with the tumor size and caloric weakness, but not with acute or insidious onset of vertigo.

- VS patients with insidious onset of vertigo symptoms tended to show increased canal weakness and postural instability.

- Further vestibular evaluation would be useful even in VS patients who do not experience acute vertigo episodes. the vestibular symptoms to the objective CDP findings in this patient population. We hypothesized that the acute vertigo symptoms the patients experience might reflect current change in vestibular nerve function, while patients who report vestibular symptoms with insidious progression reflect increased postural instability. In this study, we analyzed vestibular function tests and CDP results in VS patients and their correlation with subjective vertigo symptoms.

\section{MATERIALS AND METHODS}

The Review Board of the Gangnam Severance Hospital approved this study (IRB No. IRB3-2016-0317). All the procedures performed in the studies involving human participants were performed in accordance with the ethical standards of our institutional and/or national research committees and those of the 1964 Helsinki Declaration and its later amendments or comparable ethical standards. Since this is a retrospective study, no informed consent was obtained. The medical records of 49 patients newly diagnosed with unilateral VS from magnetic resonance imaging (MRI) findings between 2014 and 2015 were retrospectively reviewed. Twenty-two of the patients presented with vertigo symptoms, and vestibular function tests including CDP were performed in 18 patients (male:female $=8: 10$; mean age, $57.4 \pm 15.0$ years). Acute onset group included 6 patients $(33.3 \%)$ who had a history of acute episodes of vertigo within recent 3 months, and insidious onset group included 12 patients $(66.7 \%)$ who complained of vertigo/imbalance but without an acute episode. The audiovestibular evaluation included puretone audiometry, videonystagmography with bithermal caloric tests, and CDP. Subjective symptoms were evaluated using selfreport questionnaires.

\section{Subjective measurements of vertigo}

The patients indicated their feeling of dizziness on a vertigo visual analogue scale (VAS). The VAS is a 10-cm scale ranging from 0 (no dizziness) to 10 (maximum dizziness). Dizziness related handicaps in daily activities was measured using the Korean version of the Dizziness Handicap Inventory (DHI), which measures self-perceived handicaps resulting from the functional, emotional, and physical aspects of dizziness, using a 25 -item questionnaire. The DHI-total score ranges from 0 (no dizziness handicap) to 100 (maximum dizziness handicap). The DHI scores are subcategorized as functional (DHI-F, 36 points), emotional (DHI-E, 36 points), and physical (DHI-P, 28 points).

\section{MRI and tumor grading}

MRI scans were reviewed and the tumor size was measured on contrast-enhanced T1-weighted axial or coronal images. In each case, a maximum diameter was measured, and separate measurements for the internal auditory canal (IAC) and cerebello- 
pontine angle (CPA) portions were recorded. Also, tumors were graded according to Koos et al. [18]: grade I when the tumor is in the IAC, grade II when the tumor is in the CPA and $<2 \mathrm{~cm}$, grade III when the tumor is in the CPA and $2 \mathrm{~cm}$ to $4 \mathrm{~cm}$, and grade IV if the tumor is in the $\mathrm{CPA}$ and $>4 \mathrm{~cm}$.

\section{Sensory organization test}

A sensory organization test (SOT) was performed on a CDP platform (EquiTest; Neurocom, Clackamas, OR, USA). For each test, the subjects were requested to stand upright and barefoot on the platform and were instructed to look straight ahead at a picture located on the visual surround. During the SOT, the participant's task was to maintain an upright stance, as stable as possible, during three 20 seconds trials in six conditions that combined three visual conditions with two platform conditions. The subjects wore a safety harness to protect against falls. An equilibrium score (ES) was calculated by comparing the subject's anteroposterior sway during each trial to the maximal theoretical sway limits of stability, which is based on the individual's height and size of the support base. Lower sways led to a higher ES, indicating a better balance performance (a score of 100 represented no sway, while 0 indicated sway that exceeded the limits of stability, resulting in a fall). The conditions are as follows: $\mathrm{C} 1$, eyes open and the platform is stationary; $\mathrm{C} 2$, eyes closed and the platform is stationary; $\mathrm{C} 3$, visual surround moves and the platform is stationary; $\mathrm{C} 4$, eyes open and the platform is moving; $\mathrm{C} 5$, eyes closed and the platform is moving; $\mathrm{C} 6$, visual surround is moving and the platform is moving. A composite
(COMP) score is calculated by independently averaging the ES for $\mathrm{C} 1$ and $\mathrm{C} 2$, then adding them to the ES from each trial of $\mathrm{C} 3-\mathrm{C} 6$, and finally dividing this sum by the total number of trials. Each ES was adjusted to ES for C1 to identify the significance of each sensory system influencing postural control, ratio of $\mathrm{C} 2$ to $\mathrm{C} 1$ representing the somatosensory contribution to postural control (SOM), ratio of $\mathrm{C} 4$ to $\mathrm{C} 1$ representing the visual contribution, and ratio of $\mathrm{C} 5$ to $\mathrm{C} 1$ representing the vestibular contribution (VEST). The ability to rely on visual orientation cues for postural control, or visual preference, was evaluated by comparing the sway-referenced visual surround with the absence of vision $[(\mathrm{C} 3+\mathrm{C} 6) /(\mathrm{C} 2+\mathrm{C} 5)]$. Each sensory analysis ratio was considered "abnormal" when it was lower than the agespecific normative data given by the CDP manufacturer. The abnormal low SOM, visual contribution, and VEST ratio indicate that patients make poor use of each sensory system in postural stability. A low preference ratio means that patients rely on visual cues even when they are inaccurate (such as in moving visual surroundings) [19].

\section{Caloric test}

The bithermal caloric test was used, and eye movements were recorded by means of a video-based system (VisualEyes video nystagmography system; Micromedical Technologies Inc., Chatham, IL, USA). Each ear was irrigated alternately with a constant flow of water at temperatures of $30^{\circ} \mathrm{C}$ and $44^{\circ} \mathrm{C}$ and for a constant period (30 seconds). The maximum slow-phase eye velocity of nystagmus was calculated after each irrigation, and

Table 1. Clinical characteristics of patients with vestibular schwannoma presenting with vertigo symptoms

\begin{tabular}{|c|c|c|c|c|}
\hline Variable & All patients $(n=18)$ & Acute onset $(n=6)$ & Insidious onset $(n=12)$ & $P$-value \\
\hline Age (yr) & $57.4 \pm 15.0$ & $66.7 \pm 14.3$ & $52.8 \pm 13.7$ & 0.062 \\
\hline Sex (male:female) & $8: 10$ & $5: 1$ & $3: 9$ & - \\
\hline Site (right:left) & $9: 9$ & $3: 3$ & $6: 6$ & - \\
\hline \multicolumn{5}{|l|}{ Tumor size } \\
\hline Maximum size of tumor (mm) & $25.3 \pm 18.0$ & $15.9 \pm 12.4$ & $34.3 \pm 15.0$ & 0.020 \\
\hline IAC extension (no. of patients) & $16(88.9)$ & $4(66.7)$ & $12(100)$ & - \\
\hline IAC portion size & $7.3 \pm 4.3$ & $4.7 \pm 4.7$ & $8.6 \pm 3.5$ & 0.065 \\
\hline CPA portion size & $23.3 \pm 16.3$ & $10.3 \pm 10.2$ & $29.8 \pm 15.1$ & 0.012 \\
\hline Koos classification & & & & - \\
\hline I & $3(16.7)$ & $2(33.3)$ & $1(8.3)$ & \\
\hline$\|$ & $1(5.6)$ & $1(16.7)$ & 0 & \\
\hline III & $11(61.1)$ & $3(50.0)$ & $8(66.7)$ & \\
\hline IV & $3(16.7)$ & 0 & $3(25.0)$ & \\
\hline Associated symptom & & & & - \\
\hline Hearing loss in ipsilateral ear & $11(61.1)$ & $4(66.7)$ & $7(58.3)$ & \\
\hline Tinnitus & $7(38.9)$ & $3(50.0)$ & $4(33.3)$ & \\
\hline Facial palsy & $5(27.8)$ & $1(16.7)$ & $4(33.3)$ & \\
\hline \multicolumn{5}{|l|}{ PTA (dB HL) } \\
\hline Ipsilateral ear & $66.3 \pm 34.3$ & $51.5 \pm 31.8$ & $73.8 \pm 34.3$ & 0.202 \\
\hline Contralateral ear & $24.8 \pm 26.9$ & $33.5 \pm 27.1$ & $20.3 \pm 26.9$ & 0.330 \\
\hline
\end{tabular}

Values are presented as mean \pm standard deviation or number (\%).

IAC, internal auditory canal; CPA, cerebellopontine angle; PTA, pure-tone audiometry; HL, hearing level. 
Jongkees' formula was used to determine canal weakness. Canal weakness greater than $25 \%$ was defined as abnormal.

\section{Data analyses}

All statistical analyses were performed using SPSS ver. 12.0 (SPSS Inc., Chicago, IL, USA). The difference in variables between the acute onset and insidious onset groups of VS patients was tested using Pearson's chi-square test. Spearman correlation analysis was used to identify linear associations between two variables. All data are presented as mean \pm standard deviation. A $P$-value $<0.05$ was considered to reflect statistically significance.

\section{RESULTS}

The clinical characteristics of patients in the acute and chronic onset groups are described in Table 1 . The tumor size was significantly larger for the insidious onset group (mean, 34.3 \pm 15.0 $\mathrm{mm}$ ) compared to the acute onset group (mean, 15.9 \pm 12.4 $\mathrm{mm} ; P=0.020)$. Tumor extended into to the IAC in four patients $(66.7 \%)$ of acute onset group and all patients of insidious onset group. Although the tumor size for the IAC portion appeared

Table 2. Comparison of subjective symptom severity and vestibular function tests in VS patients with acute onset or insidious onset of vertigo symptoms

\begin{tabular}{lcccc}
\hline Variable & $\begin{array}{c}\text { All patients } \\
(\mathrm{n}=18)\end{array}$ & $\begin{array}{c}\text { Acute onset } \\
(\mathrm{n}=6)\end{array}$ & $\begin{array}{c}\text { Insidious } \\
\text { onset }(\mathrm{n}=12)\end{array}$ & $P$-value \\
\hline VAS & $4.6 \pm 2.4$ & $3.5 \pm 3.0$ & $5.0 \pm 2.2$ & 0.318 \\
DHI & & & & \\
P subscale & $10.3 \pm 7.5$ & $10.0 \pm 9.4$ & $10.4 \pm 7.2$ & 0.627 \\
E subscale & $14.7 \pm 11.4$ & $15.0 \pm 16.7$ & $14.8 \pm 9.7$ & 0.978 \\
\hline F subscale & $18.3 \pm 12.2$ & $18.0 \pm 16.5$ & $18.4 \pm 11.2$ & 0.958 \\
Total score & $43.4 \pm 30.0$ & $43.0 \pm 42.3$ & $43.6 \pm 26.6$ & 0.980 \\
Caloric & $63.4 \pm 34.2$ & $30.8 \pm 6.2$ & $77.9 \pm 31.1$ & 0.014 \\
weakness (\%) & & & & \\
CDP parameter & & & & \\
ES C1 & $92.9 \pm 2.3$ & $92.6 \pm 2.9$ & $93.1 \pm 2.1$ & 0.663 \\
ES C2 & $91.4 \pm 2.4$ & $90.8 \pm 3.4$ & $91.8 \pm 1.8$ & 0.394 \\
ES C3 & $88.3 \pm 3.7$ & $90.9 \pm 4.1$ & $87.0 \pm 2.9$ & 0.074 \\
ES C4 & $72.8 \pm 15.7$ & $67.5 \pm 24.7$ & $75.4 \pm 9.1$ & 0.334 \\
ES C5 & $45.2 \pm 19.8$ & $48.4 \pm 25.2$ & $43.6 \pm 17.5$ & 0.644 \\
ES C6 & $39.4 \pm 21.7$ & $45.4 \pm 24.8$ & $36.5 \pm 20.5$ & 0.431 \\
COMP & $65.9 \pm 11.0$ & $68.7 \pm 13.1$ & $64.6 \pm 10.1$ & 0.474 \\
SOM & $1.0 \pm 0.0$ & $1.0 \pm 0.0$ & $1.0 \pm 0.0$ & 0.616 \\
VIS & $0.8 \pm 0.2$ & $0.7 \pm 0.3$ & $0.8 \pm 0.1$ & 0.368 \\
VEST & $0.5 \pm 0.2$ & $0.5 \pm 0.3$ & $0.5 \pm 0.2$ & 0.641 \\
PREF & $0.9 \pm 0.1$ & $1.0 \pm 0.1$ & $0.9 \pm 0.1$ & 0.138 \\
\hline
\end{tabular}

Values are presented as mean \pm standard deviation.

VS, vestibular schwannoma; VAS, visual analogue scale; DHI, Dizziness Handicap Inventory; P, physical; E, emotional; F, functional; CDP, computerized dynamic posturography; ES, equilibrium score; C, condition; COMP, composition; SOM, somatosensory; VIS, visual; VEST, vestibular; PREF, preference. larger for the insidious onset group, the difference was not statistically significant. The tumor size for the CPA portion was larger for the insidious onset group $(P=0.012)$. Acute onset group included grade I, II and III, and insidious onset included one case of grade I and others were graded III and IV according to Koos classifications. Most commonly associated symptom was hearing loss, followed by tinnitus and facial palsy. Hearing thresholds in the ipsilateral ears were worse in the insidious onset group, but the difference was not statistically significant.

Subjective perception of symptoms severity as measured by VAS ranged from mild to severe (1-10), and did not show significant difference between the acute onset and insidious onset groups $(P=0.318)$. Likewise, the DHI total score or subscale scores (DHI-P, E, and F) did not differ between two groups $(P>0.05)$. Caloric weakness was noted in 13 of all patients (72.2\%). All patients showed decreased ES for $\mathrm{C} 5$ and $\mathrm{C} 6$, and resulting VEST and COMP scores were also decreased, supporting that postural instability is prevalent in these patients. When the acute onset and insidious onset groups were compared, the VAS and DHI scores did not show significant difference. The insidious onset group had higher rate of caloric weakness $(100 \%)$, and caloric weakness was greater $(P=0.014)$ than the acute onset group (Table 2).

Correlation between postural instability measured as COMP score and other associated factors was examined (Table 3). The COMP scores were not correlated with VAS or DHI scores. Tumor sizes (the maximum diameter, IAC or CPA portion sizes) did not show significant correlation with COMP scores. Fig. 1 shows the box-plots to compare variables related to postural instability between the acute onset and insidious onset groups. The ES for C5 and C6,VEST and COMP scores were generally lower for the insidious onset group compared to acute onset group, but the difference was not statistically significant (Fig. 1).

\section{DISCUSSION}

Vestibular symptoms in VS patients are widely variable. Although the VS is a slowly-growing tumor, some patients may experience acute onset of severe vertigo, while others develop subtle or no symptoms. Maximum tumor size, nerve of origin,

Table 3. The correlation between the COMP scores and associated factors in VS patients

\begin{tabular}{lcc}
\hline Variable & COMP & $\begin{array}{c}\text { Spearman correlation } \\
\text { coefficient }\end{array}$ \\
\hline Tumor size & $P=0.632$ & $R=-0.121$ \\
VAS & $P=0.547$ & $R=0.176$ \\
DHI total score & $P=0.753$ & $R=0.093$ \\
Canal weakness & $P=0.167$ & $R=-0.408$ \\
\hline
\end{tabular}

COMP, composition; VS, vestibular schwannoma; VAS, visual analogue scale; DHI, Dizziness Handicap Inventory. 

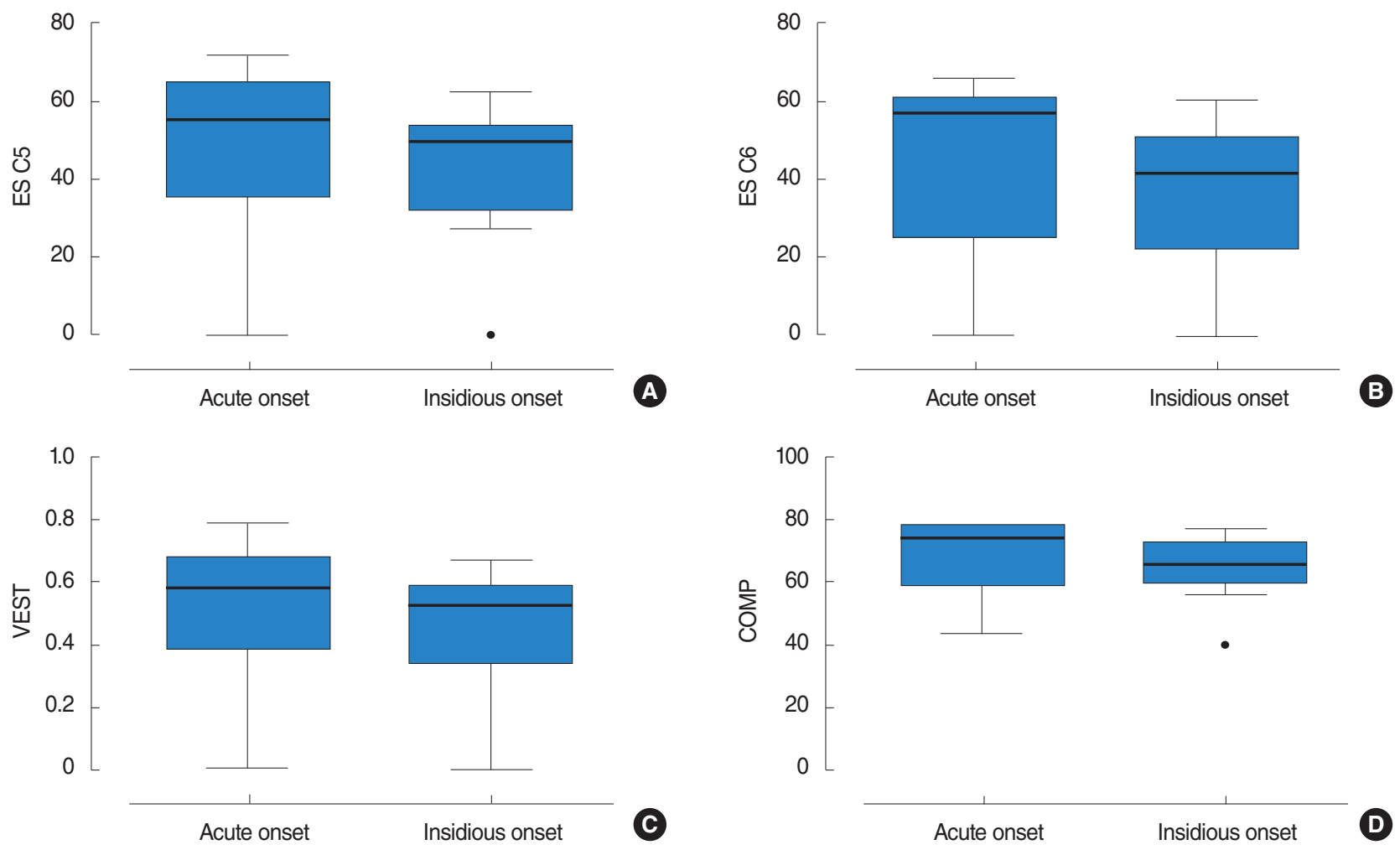

Fig. 1. Box-plots of postural instability-related variables: (A) ES C5, (B) ES C6, (C) VEST, and (D) COMP scores for the acute onset and insidious onset groups of vestibular schwannoma patients. Each box represents the lower (the 25th percentile) to the upper range (the 75th percentile) and contains the median value shown as a line across. The "whiskers" of the box extend to the farthest points that are not outliers (i.e., they are within 1.5 times the interquartile range of the 25th and 75th percentiles). Outside values are depicted as circle lines. ES, equilibrium score; C, condition; VEST, vestibular; COMP, composition.

associated cochlear symptoms and objective vestibular function test results have been investigated for their correlation of symptom severity of vertigo and imbalance. However, little is known about acute onset of symptoms in VS patients. It has been surmised that acute change in tumor size, such as tumor growth or intratumoral hemorrhage or cystic change would compress the vestibular nerves or cerebellum leading to acute vestibular symptoms. Direct compression of the vestibular nerves would lead to demyelination, and also decreased microcirculation would damage the neural structures. In addition, metabolic changes caused by tumoral secretions from VS have been related to cochlear damage causing sensorineural hearing loss, and similar effects on vestibular organs may contribute to their abrupt or gradual loss of function [20].

In this study, we aimed to investigate whether tumor characteristics and vestibular functional status of vestibular system and postural control are related to acute onset or insidious onset of vertigo. Our results showed that the maximum size of tumor and the IAC and CPA portion sizes were larger, hearing impairment was more common, and canal weakness was greater in patients with insidious onset of vestibular symptoms. It can be suggested that acute onset of vertigo is not related to tumor size.
Sudden change in vestibular sensory input, possibly by mechanical or biochemical damage can be postulated as possible explanation for acute bouts of vertigo. Considering that caloric weakness was more evident in the insidious onset group, acute onset of symptoms may reflect acute deterioration of residual vestibular function. While the insidious onset group showed greater caloric weakness, gradual vestibular compensation in these patients might explain the lack of difference in SOT parameters compared to acute onset vertigo group. However, it remains a question whether the direct compression of the nerves can be deduced from tumor size measurements, and a longitudinal follow-up study of VS patients with IAC involvement to correlate changes in tumor dimensions in the IAC and development of acute vertigo symptoms would be informative. Another explanation is that the proximity of the tumor to the inner ear in VS patients with IAC involvement would facilitate diffusion of metabolites that cause cochlear and vestibular damage. However, VS-secreted metabolites have been identified only recently, and their possible effect on vestibular organs are still unknown.

The CDP is widely employed, and the SOT paradigm provides essential information regarding a patient's ability to maintain stable posture. While static balance tests such as the Rom- 
berg test or standing on a foam are simpler to perform in a clinic, the CDP offers the obvious advantage to assess sensory integration in sensory conflict situations, by altering the visual and proprioceptive inputs in six different conditions. Previous studies have reported that the ES for SOT conditions C5 and C6 are related to canal paresis as measured by caloric or rotational chair tests in the VS patients [6] and whether they complain of vertigo symptoms [16]. Gait disturbance was noted in patients with small tumors, especially while walking with eyes closed [21]. Postural instability impacts daily activities, and it has been shown that the vestibular symptoms correlate with prospective working disability in VS patients [8]. These findings support implantation of CDP in vestibular function evaluation of VS patients. In our analysis, we focused on ES for C5 and 6, COMP and VEST scores: the C5 and C6 conditions test the patients' ability to maintain posture relying on vestibular sensory inputs, and VEST and COMP scores takes ES for C5 and/or C6 into consideration. Dynamic postural instability measured by these parameters did not differ between patients with acute or insidious symptoms. Interestingly, there was a tendency of increased postural instability in patients with insidious onset, but the difference was not statistically significant. With slow growth of tumors and gradual deterioration of vestibular inputs, central compensation would take place and alleviate expected acute symptoms, and it is plausible that some patients would experience intermittent or subtle imbalance. Indeed, many patients would vaguely describe their symptoms as vertigo or imbalance that occurs "sometimes, but not always" over some period of time. Since many are referred after diagnosis of VS, the patients were already apprehensive about their conditions and sometimes they would not bring up subtle vertigo complaints unless asked. During initial interviews with patients who visited our tertiary-referral clinic, we asked specific questions regarding hearing status and vertigo symptoms. Our results show even these nonspecific symptoms should alert the clinician to suspect postural instability and consider CDP testing.

Evaluation of vestibular function and posture control in VS patients is important because their prominent effect on patients' quality of life. In patients with untreated VS, vertigo symptoms negatively affected quality of life, while hearing symptoms were less damaging [22]. Also, vertigo symptoms were more associated with disability affecting VS patients' working status after treatment than hearing problems [8]. While hearing loss and facial nerve dysfunction can be measured easily, vestibular tests often require more expertise and attention. Furthermore, clinicians may be focused to utilize vestibular function tests as tools to estimate tumor size, nerve of origin and treatment outcomes, which are essential in treatment planning. In addition to these pertinent information, patients' performance in posture control should also be considered as an important index of their general well-being.

Limitations of this study include that only VS patients with subjective vertigo symptoms were evaluated for postural instability. About half of the newly diagnosed VS patients did not have vestibular symptoms and CDP was not tested, and further vestibular evaluation would have been valuable to confirm subclinical vestibular dysfunction. In addition to the conventional caloric test, VEMP and vHIT would have provided more comprehensive description of vestibular function status, and their correlation with postural instability remains to be explored. Prospective longitudinal studies on larger groups of VS patients would provide more detailed information regarding postural instability and their symptom correlation.

This study was aimed to examine factors associated with subjective vestibular symptoms and postural instability in VS patients. The patients with insidious onset of vertigo symptoms tended to show increased canal weakness and postural instability. Thus, clinicians should consider that postural instability is likely present even in patients who do not complain of acute vertigo, and appropriate counseling should be discussed with the patients.

\section{CONFLICT OF INTEREST}

No potential conflict of interest relevant to this article was reported.

\section{ACKNOWLEDGMENTS}

This research was supported by the Basic Science Research Program of the National Research Foundation of Korea (NRF), funded by the Ministry of Education, Science and Technology (Grant No. 2016R1A2B1012521 to EJS), Republic of Korea and by a faculty research grant from Yonsei University College of Medicine (6-2016-0092) Seoul, Korea. The funders played no role in the study design, data collection or analysis, the decision to publish, or manuscript preparation.

\section{REFERENCES}

1. Kentala E, Pyykko I. Clinical picture of vestibular schwannoma.Auris Nasus Larynx. 2001 Jan;28(1):15-22.

2. Andersen JF, Nilsen KS, Vassbotn FS, Moller P, Myrseth E, Lund-Johansen $\mathrm{M}$, et al. Predictors of vertigo in patients with untreated vestibular schwannoma. Otol Neurotol. 2015 Apr;36(4):647-52.

3. Hoffmann CP, Seigle B, Frere J, Parietti-Winkler C. Dynamical analysis of balance in vestibular schwannoma patients. Gait Posture. 2017 May;54:236-41.

4. Day AS, Wang CT, Chen CN, Young YH. Correlating the cochleovestibular deficits with tumor size of acoustic neuroma. Acta Otolaryngol. 2008 Jul;128(7):756-60.

5. Gouveris H,Akkafa S, Lippold R, MannW. Influence of nerve of origin and tumor size of vestibular schwannoma on dynamic posturog- 
raphy findings. Acta Otolaryngol. 2006 Dec;126(12):1281-5

6. Gouveris H, Helling K, Victor A, Mann W. Comparison of electronystagmography results with dynamic posturography findings in patients with vestibular schwannoma. Acta Otolaryngol. 2007 Aug;127(8): 839-42.

7. Morrison GA, Sterkers JM. Unusual presentations of acoustic tumours. Clin Otolaryngol Allied Sci. 1996 Feb;21(1):80-3.

8. Breivik CN, Nilsen RM, Myrseth E, Finnkirk MK, Lund-Johansen M. Working disability in Norwegian patients with vestibular schwannoma: vertigo predicts future dependence. World Neurosurg. 2013 Dec;80(6):e301-5.

9. Dayal M, Perez-Andujar A, Chuang C, Parsa AT, Barani IJ. Management of vestibular schwannoma: focus on vertigo. CNS Oncol. 2013 Jan;2(1):99-104.

10. Tranter-Entwistle I, Dawes P, Darlington CL, Smith PF, Cutfield N. Video head impulse in comparison to caloric testing in unilateral vestibular schwannoma. Acta Otolaryngol. 2016 Nov;136(11):1110-4.

11. Batuecas-Caletrio A, Santa Cruz-Ruiz S, Munoz-Herrera A, PerezFernandez N. The map of dizziness in vestibular schwannoma. Laryngoscope. 2015 Dec;125(12):2784-9.

12. Bergenius J, Magnusson M. The relationship between caloric response, oculomotor dysfunction and size of cerebello-pontine angle tumours. Acta Otolaryngol. 1988 Nov-Dec;106(5-6):361-7.

13. Stipkovits EM, Van Dijk JE, Graamans K. Electronystagmographic changes in patients with unilateral vestibular schwannomas in relation to tumor progression and central compensation. Eur Arch Otorhinolaryngol. 1999 Apr;256(4):173-6.

14. Kim HJ, Park SH, Kim JS, Koo JW, Kim CY, Kim YH, et al. Bilateral- ly abnormal head impulse tests indicate a large cerebellopontine angle tumor. J Clin Neurol. 2016 Jan;12(1):65-74.

15. Monsell EM, Furman JM, Herdman SJ, Konrad HR, Shepard NT. Computerized dynamic platform posturography. Otolaryngol Head Neck Surg. 1997 Oct;117(4):394-8.

16. Gouveris H, Stripf T, Victor A, Mann W. Dynamic posturography findings predict balance status in vestibular schwannoma patients. Otol Neurotol. 2007 Apr;28(3):372-5.

17. Ribeyre L, Frere J, Gauchard G, Lion A, Perrin P, Spitz E, et al. Preoperative balance control compensation in patients with a vestibular schwannoma: does tumor size matter? Clin Neurophysiol. 2015 Apr;126(4):787-93.

18. Koos WT, Spetzler RF, Bock FW. Microsurgery of cerebellopontine angle tumors. In: Koos WT, Bock FW, Spetzler RF, Ammerman B, editors. Clinical microneurosurgery. Stuttgart: Georg Thieme; 1976. p. 91-112.

19. Neurocom International Inc. EquiTest system operator's manual version 8.2. Clackamas: Neurocom International Inc.; 2004.

20. Dilwali S, Landegger LD, Soares VY, Deschler DG, Stankovic KM. Secreted factors from human vestibular schwannomas can cause cochlear damage. Sci Rep. 2015 Dec;5:18599.

21. Yin M, Ishikawa K, Omi E, Saito T, Itasaka Y,Angunsuri N. Small vestibular schwannomas can cause gait instability. Gait Posture. 2011 May;34(1):25-8.

22. Myrseth E, Moller P,Wentzel-Larsen T, Goplen F, Lund-Johansen M. Untreated vestibular schwannomas: vertigo is a powerful predictor for health-related quality of life. Neurosurgery. 2006 Jul;59(1):6776. 